\title{
Numerical Flow Analysis in a Rotating Square Duct and a Rotating Curved-Duct*
}

\author{
JE HYUN BAEK ${ }^{\dagger}$ and CHANG HWAN KO \\ School of Environmental Engineering, POSTECH, San 31, HyoJa Dong, Namgu, Pohang, 790-784 Korea
}

(Received 24 April 1998; In final form 7 July 1998)

\begin{abstract}
A numerical study is conducted on the fully-developed laminar flow of an incompressible viscous fluid in a square duct rotating about a perpendicular axis to the axial direction of the duct. At the straight duct, the rotation produces vortices due to the Coriolis force. Generally two vortex cells are formed and the axial velocity distribution is distorted by the effect of this Coriolis force. When a convective force is weak, two counter-rotating vortices are shown with a quasi-parabolic axial velocity profile for weak rotation rates. As the rotation rate increases, the axial velocity on the vertical centreline of the duct begins to flatten and the location of vorticity center is moved near to wall by the effect of the Coriolis force. When the convective inertia force is strong, a double-vortex secondary flow appears in the transverse planes of the duct for weak rotation rates but as the speed of rotation increases the secondary flow is shown to split into an asymmetric configuration of four counter-rotating vortices. If the rotation rates are increased further, the secondary flow restabilizes to a slightly asymmetric double-vortex configuration. Also, a numerical study is conducted on the laminar flow of an incompressible viscous fluid in a $90^{\circ}$-bend square duct that rotates about axis parallel to the axial direction of the inlet. At a $90^{\circ}$-bend square duct, the feature of flow by the effect of a Coriolis force and a centrifugal force, namely a secondary flow by the centrifugal force in the curved region and the Coriolis force in the downstream region, is shown since the centrifugal force in curved region and the Coriolis force in downstream region are dominant respectively.
\end{abstract}

Keywords: Rotating channel, Numerical analysis, Coriolis force, Centrifugal force, Secondary flow, Laminar

\section{INTRODUCTION}

The study of a flow in a rotating duct is of interest in fluid mechanics. The earliest work on this subject consisted of theoretical investigations of the weak rotation case for laminar flow in circular pipes. Barua (1954) showed that for weak rotations the secondary flow in the duct consisted of a counterrotating double-vortex. A short time later, Benton (1956) calculated the secondary flow and the

\footnotetext{
* This paper was originally presented at ISROMAC-7.

${ }^{\dagger}$ Corresponding author. Tel.: 82562 279 2168. Fax: 825622793199. E-mail: jhbaek@postech.ac.kr.
} 
distortion of the axial velocity profile by the effect of Earth's rotation. And Hart (1971) proved experimentally and theoretically that at relatively rapid rotation rates an instability exists in the form of longitudinal roll cells. At higher rotation rates, he showed that the flow restabilizes and the gradient of the axial velocity along the axis of rotation in the interior of the duct is zero. Johnston et al. (1972) and Lezius and Johnston (1976) studied instabilities for laminar and turbulent flows in a rotating duct. Speziable (1982) and Kheshgi and Scriven (1985) studied the feature of laminar flow in rotating square ducts using the numerical method.

In this paper, a numerical study is conducted on the three-dimensional, steady and fully-developed laminar flow of an incompressible viscous fluid in a straight square duct rotating about a perpendicular axis to the axial direction of the duct and in a $90^{\circ}$ bend square duct rotating about an axis parallel to the axial direction of the entrance region. Figures 1 and 2 show the schematics of a straight square duct and a curved duct rotating about a constant axis.

When the straight square-duct system rotates about an axis not parallel to that of the duct, that flow is not rectilinear. The Coriolis force due to the rotation throws the fast moving fluid in the duct core towards the direction of the force. In general, two vortex cells in the cross-section are then formed and the axial velocity profile is distorted. When convective inertia become important, the equation of motion become nonlinear and the possibility of multiple steady flow states aries. Indeed, under certain conditions a four-vortex flow is formed.

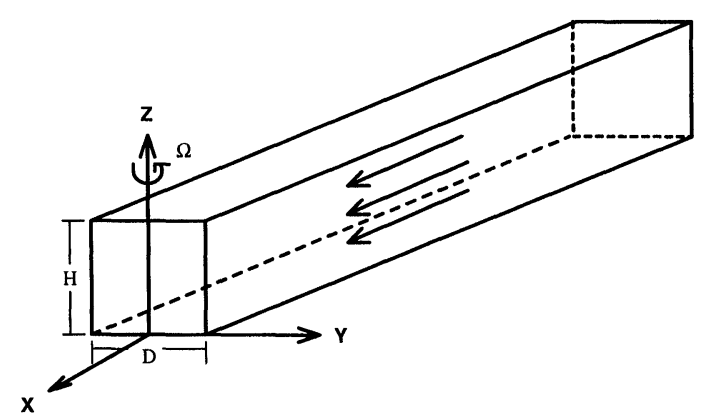

FIGURE 1 Scheme of a rotating straight square duct.
When the $90^{\circ}$-bend square duct (entrance length $=45 D$, exit length $=30 D$, mean radius of curvature $=2.3 \mathrm{D}$ ) rotates about an axis parallel to the axial direction of the entrance region, the complex flow feature of the effects of the Coriolis force and the centrifugal force is shown. A secondary flows by the centrifugal force in the curved region and the Coriolis force in the downstream region are shown, since the centrifugal force in the curved region and the Coriolis force in the downstream region dominates flow respectively. Axial flow velocity profile is distorted by those effects of the centrifugal force and the Coriolis force.

In this numerical analysis, SIMPLE (SemiImplicit Method for Pressure Linked Equations) algorithm is used to solve the full Navier-Stokes system, cast in a rotating frame of reference, for straight and curved ducts. In the case of a straight duct, a periodic condition $(V(x=0)=V(x=L))$ is used as a boundary condition. Therefore twodimensional flow is observed at the converged solution. In the case of a curved duct, Neumann condition $(\partial V / \partial z=0)$ is used at the exit region. In this paper, the flow is analysed for a variety of Reynolds number, Ekman number and Rossby number. The flows in the straight and curved ducts are compared with each other.

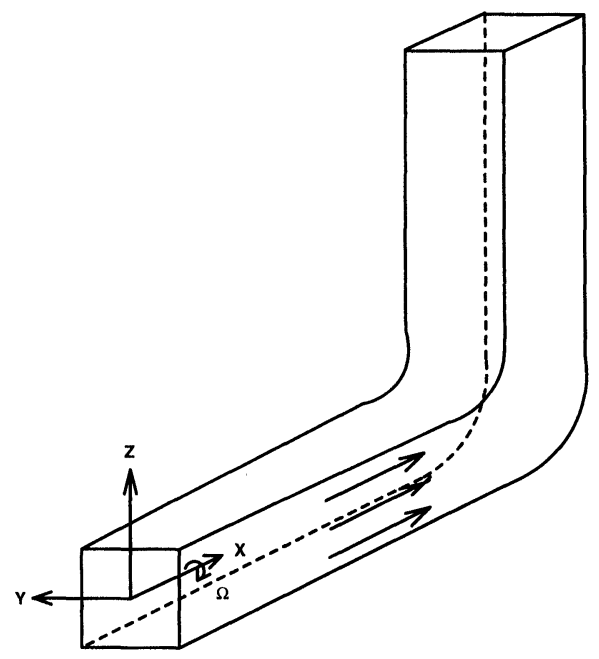

FIGURE 2 Scheme of a rotating curved square duct. 


\section{GOVERNING EQUATION}

The Navier-Stokes equations in the frame of reference rotating with the duct are

$$
\begin{gathered}
\frac{\partial \vec{V}}{\partial t}+(\vec{V} \cdot \nabla) \vec{V}+\vec{\Omega} \times(\vec{\Omega} \times \vec{r})+2 \vec{\Omega} \times \vec{V} \\
=-\frac{1}{\rho} \nabla p+\nu \nabla^{2} \vec{V} \\
\nabla \cdot \vec{V}=0 .
\end{gathered}
$$

When $\vec{\Omega}$ is equal to $\Omega \vec{k}$ and the centrifugal term is combined with the thermodynamic pressure in the modified pressure $P=p-\frac{1}{2} \rho \Omega^{2}\left(x^{2}+y^{2}\right)$, the dimensionless Navier-Stokes system becomes

$$
\begin{gathered}
\frac{\partial \vec{V}^{*}}{\partial t^{*}}+R o\left(\vec{V}^{*} \cdot \nabla^{*}\right) \vec{V}^{*}+2 \vec{k} \times \vec{V}^{*} \\
=-\nabla^{*} P^{*}-E k \nabla^{* 2} \vec{V}^{*} \\
\nabla^{*} \cdot \vec{V}^{*}=0 .
\end{gathered}
$$

Here $\mathrm{Ro}=U / \vec{\Omega} D$ is Rossby number and Ek $=\nu /$ $\vec{\Omega} D^{2}$ is Ekman number. The position of the axis of rotation does not have an effect on the flow as shown in Eq. (3), whereas the rate of rotation $\vec{\Omega}$ has an effect on the flow through Coriolis force.

\section{RESULT AND DISCUSSION}

\subsection{Straight Square Duct}

The problem to be considered is that of the laminar flow of an incompressible viscous fluid in a straight square duct that is subjected to a constant rotation $\vec{\Omega}$. In the absence of rotation the fully-developed velocity field is of the unidirectional form. When the axial pressure gradient $\partial P / \partial x=-\bar{G}$ is constant, the fully-developed velocity field is determined from the Poisson equation:

$$
\nabla^{2} u=-\frac{\bar{G}}{\mu} .
$$

This flow yields the quasi-parabolic profile for the axial velocity. However, for non-zero rotation rates, the flow field becomes three-dimensional, where exist a secondary flow. In the system with rotation, the governing equation takes the form of the Eqs. (3) and (4). Then the axial velocity profile is distorted from parabolic velocity profile. In Eq. (3), the Coriolis term serves as the driving mechanism for the creation of secondary flow in a rotating duct.

When the duct rotates slowly, the effect of a slight amount of rotation serves as a perturbation for the flow through rotating duct. A small amount of rotation forces a secondary flow with two counter-rotating vortices. When the duct rotates rapidly, the flow in the duct interior is dominated by the Coriolis force and surrounded by horizontal Ekman layers along the channel roof and floor, and vertical double layers along the left and right walls.

When the effect of convective inertia term $\operatorname{Ro}\left(\vec{V}^{*} \cdot \nabla^{*}\right) \vec{V}^{*}$ is strong, a double-vortex secondary flow appears in the transverse planes of the duct for weak rotation rates but as the speed of rotation increases, the secondary flow is shown to be splitted into an asymmetric configuration of four vortices. If the rotation rates are increased further, the secondary flow restabilizes to a asymmetric double-vortex configuration.

When the effect of convective inertia term is weak $(\mathrm{Re}=10)$, the progression of flow fields with Ekman number is illustrated in Fig. 3 by the vector fields and axial velocity contours in the transverse plane. Regardless of Ekman number, it is shown that the flow contains a pair of counter-rotating vortices in the plane perpendicular to the axis of rotation. And the center of vortex moves to upper and lower wall, as the rate of rotation increases. Because the rate of rotation is small at high Ekman number $(E k=0.1)$, Coriolis force does not give a substantial effect on the flow. Therefore the axial velocity profile shown in Fig. 4 has quasi-parabolic velocity distribution. But at low Ekman number $(E k=0.001)$, the Coriolis force dominates flow in the duct interior except for thin viscous boundary layers (Ekman boundary layer and vertical double boundary layer).

When Ekman number decreases, at the near region of the upper or lower wall the value of $v_{\max } / u_{\max }$ are $0.0494,0.228$ and 0.281 for $\mathrm{Ek}=0.1$, 


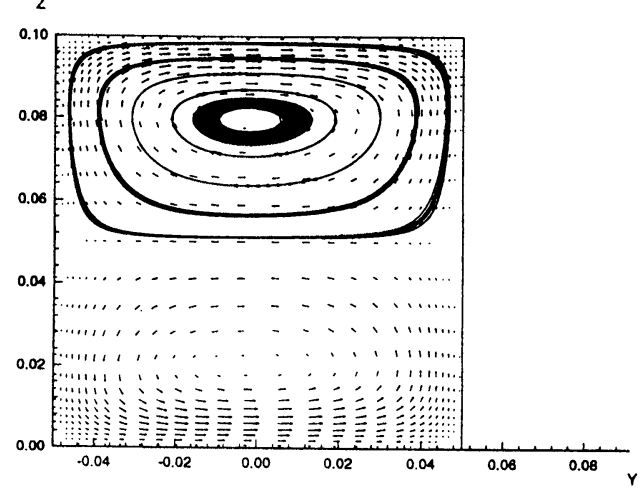

$E k=0.1$

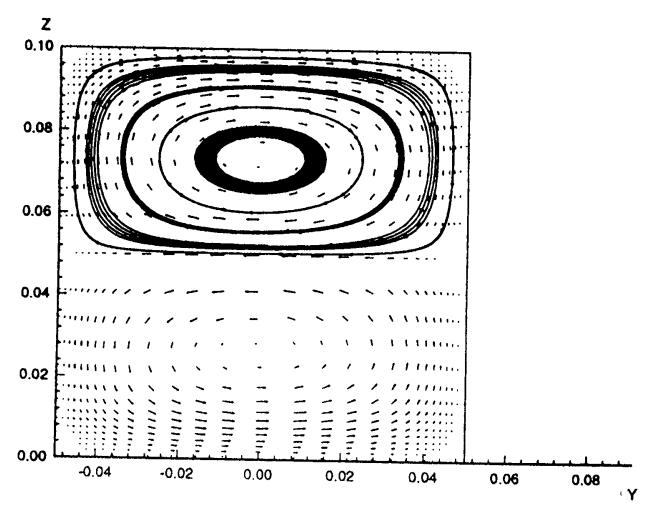

$E k=0.01$

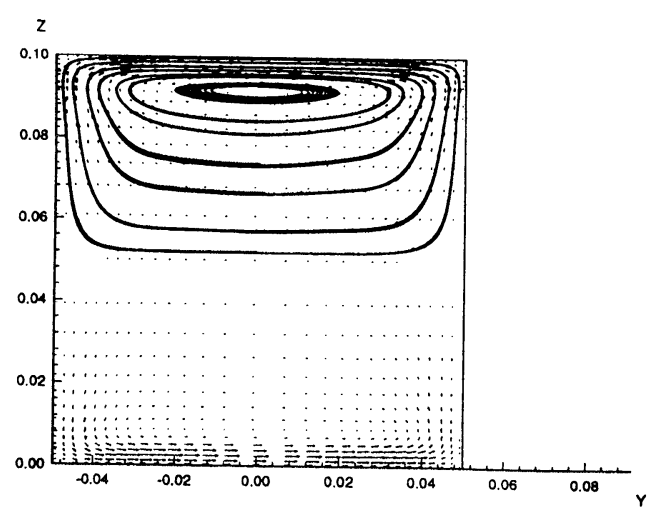

$E k=0.001$ (b)

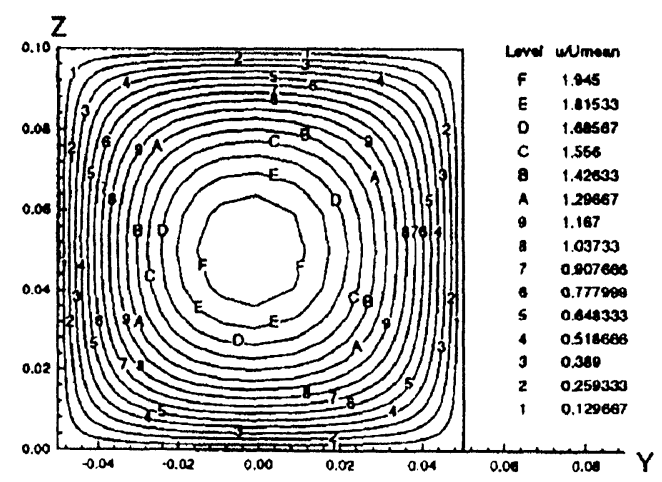

$\mathrm{Ek}=0.1$

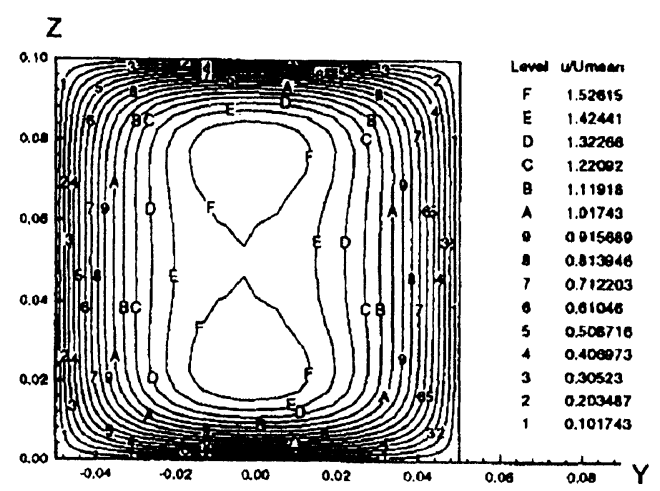

$\mathrm{Ek}=0.01$

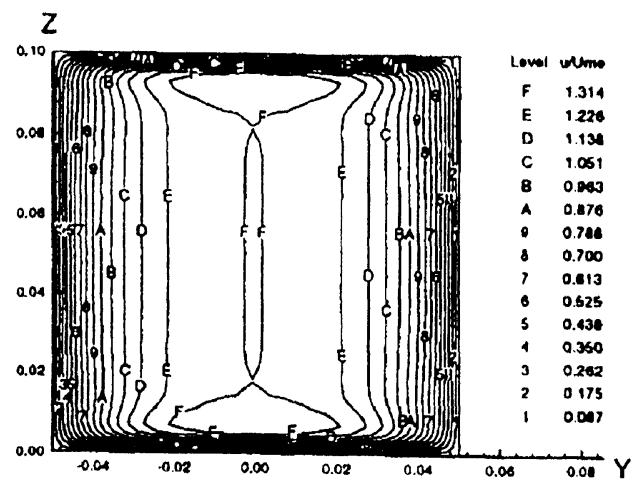

$\mathrm{Ek}=0.001$

FIGURE 3 (a) Secondary flow in rotating square duct for three Ekman number at $\mathrm{Re}=10$; (b) Axial velocity contours for three Ekman number at $\mathrm{Re}=10$. 


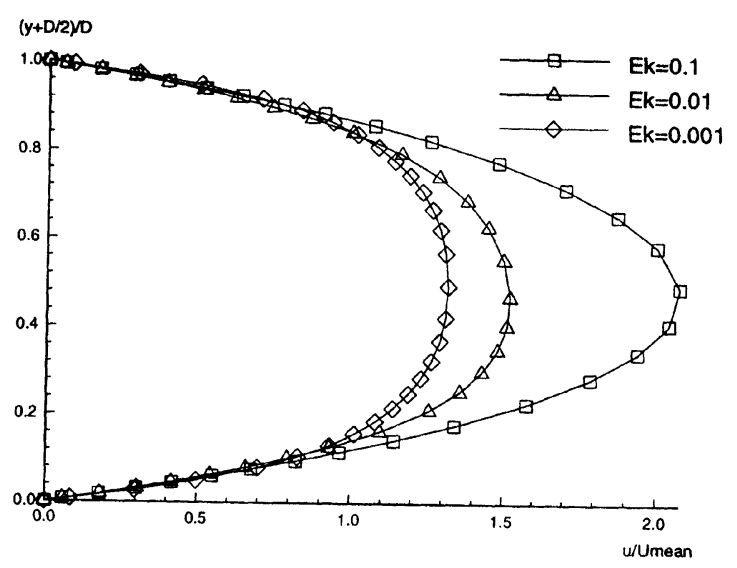

a)

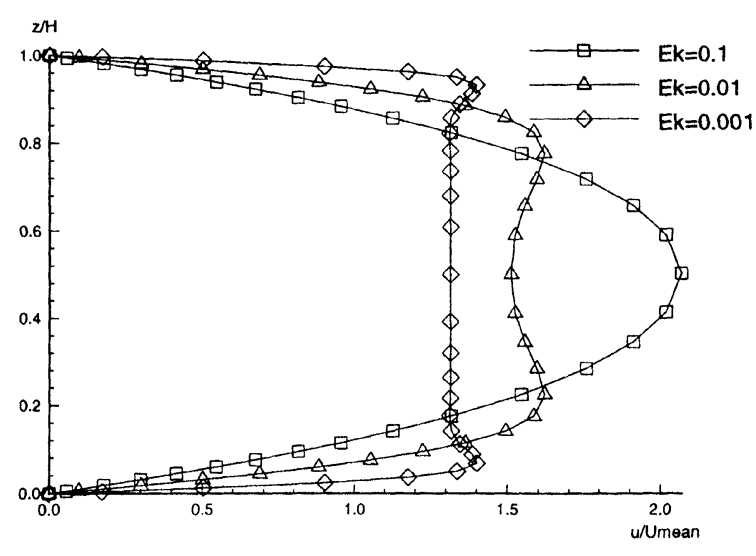

b)

FIGURE 4 Axial velocity profile in a rotating square duct for $\mathrm{Re}=10$ : (a) along the horizontal centerline of the duct; (b) along the vertical centerline of the duct.

0.01 and 0.001 respectively. At high Ekman number, the secondary flow is weak but at low Ekman number, the magnitude of the secondary flow is comparable to axial velocity. As the speed of rotation increases, the strength of the secondary flow becomes larger. At low Ekman number $(E k=0.001)$ the maxima in the axial velocity are within the Ekman boundary layer and the flow has nearly uniform axial velocity profile in the vertical planes of the duct as can be seen in Fig. 3(b). When the effect of convective inertia force is weak, Fig. 4 shows the axial velocity profiles in the horizontal and the vertical centreplane for various Ekman numbers. When Ekman number is 0.1 , the axial velocity profile along the horizontal centreline is quasi-parabolic. But those become blunt as Ekman number decreases. In Fig. 4(b) the axial velocity along the vertical centreline of the duct is symmetric and begins to flatten in the core region of the duct with the increase of rotation rate. When Ekman number is 0.001 , the gradient of the axial velocity along the vertical centreline is zero in the core region and the peaks of the axial velocity is located in the Ekman boundary layer.

When the effect of convective inertia is not small $(\mathrm{Re}=500)$, the progression of flow fields with Ekman number is illustrated in Figs. 5 and 6. When convective inertia plays a significant role, more complicated flow evolves, that is, the unstable flow with a four-vortex exists in the duct interior.

When Ekman number is high $(\mathrm{Ek}=0.1)$, a doublevortex secondary flow appears in the transverse planes of the duct. But as the speed of rotation increases, the secondary flow with two vortices split into a four-vortex secondary flow. If the rotation rate is increased further, the secondary flow restabilizes to a slightly asymmetric double-vortex configuration. The unstable flow with a four-vortex results from a disparity in the symmetry of the convective and the Coriolis terms. In Fig. 6(a), the axial velocity profile in the horizontal centreplane of the duct has asymmetric form and the peak of the axial velocity is shifted in the direction of Coriolis force. The axial velocity profile on the vertical centreplane of the duct has a symmetric form and flattens with the increasing of the rate of rotation. But the unstable flow with a four-vortex $(\mathrm{Ek}=0.01)$ shows three peaks in velocity profile. As the rate of rotation increases further, the axial velocity profile on the vertical centreplane shows two peaks in the near region of the upper and lower walls with the plateau in core region. Near the upper and lower wall, the values of $v_{\max } / u_{\max }$ are $0.033,0.12$ and 0.28 for $\mathrm{Ek}=0.1,0.01$ and 0.001 respectively. The secondary flow distorts substantially the axial velocity profile even though the magnitude of secondary flow is small as shown in 
(a)

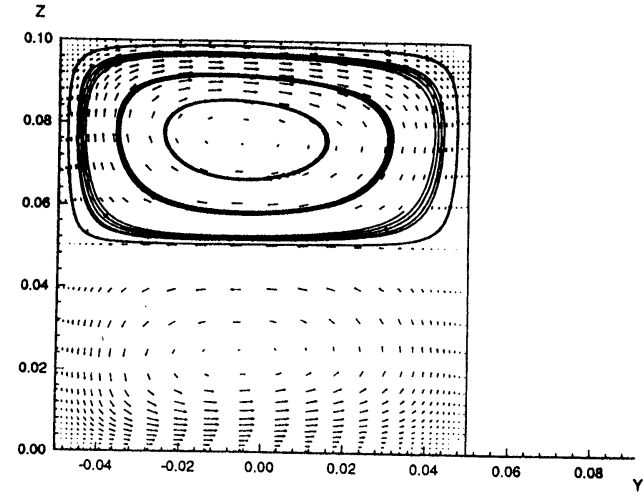

$E k=0.1$

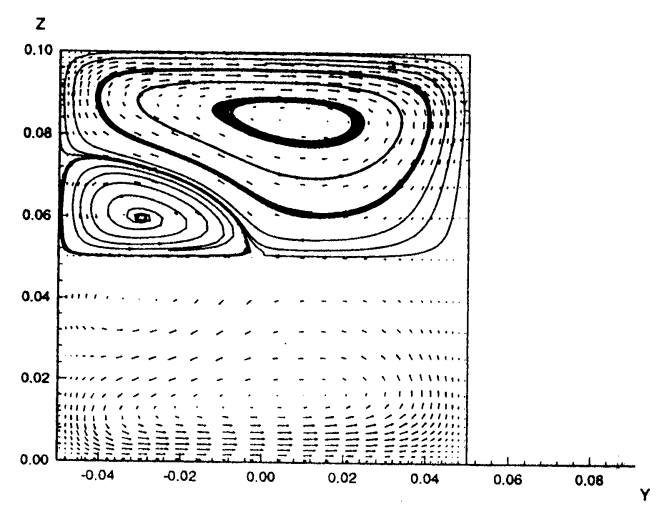

$\mathrm{Ek}=0.01$

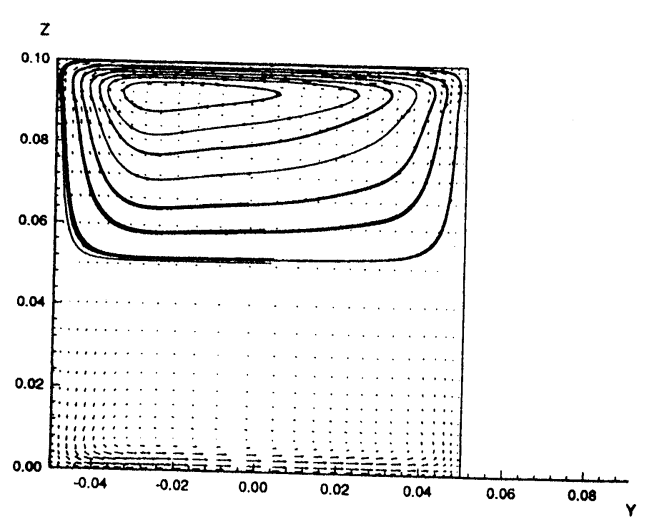

$\mathrm{Ek}=0.00075$ (b)

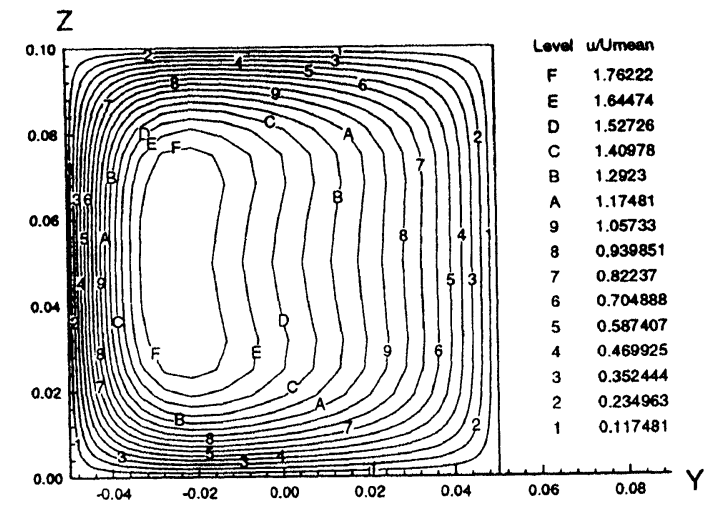

$\mathrm{Ek}=0.1$

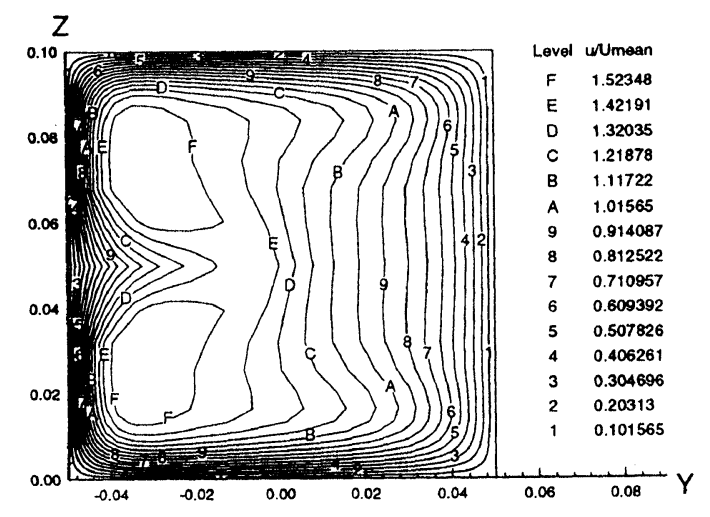

$\mathrm{Ek}=0.01$

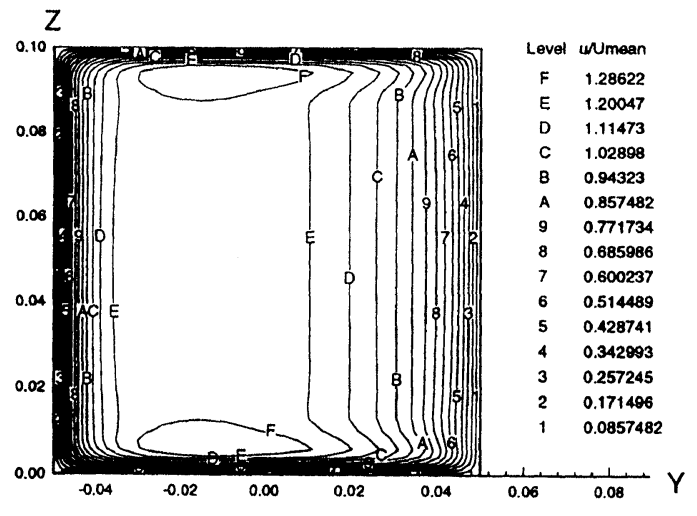

$E k=0.00075$

FIGURE 5 (a) Secondary flow in rotating square duct for three Ekman number at $\mathrm{Re}=500$; (b) Axial velocity contours for three Ekman number at $\mathrm{Re}=500$. 


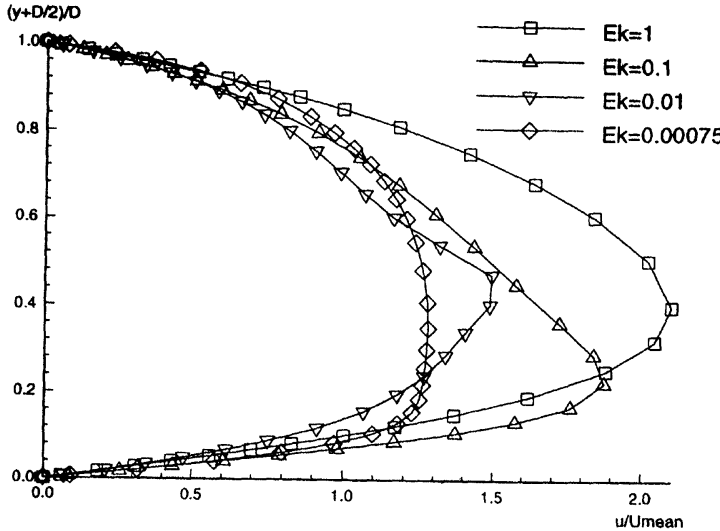

a)

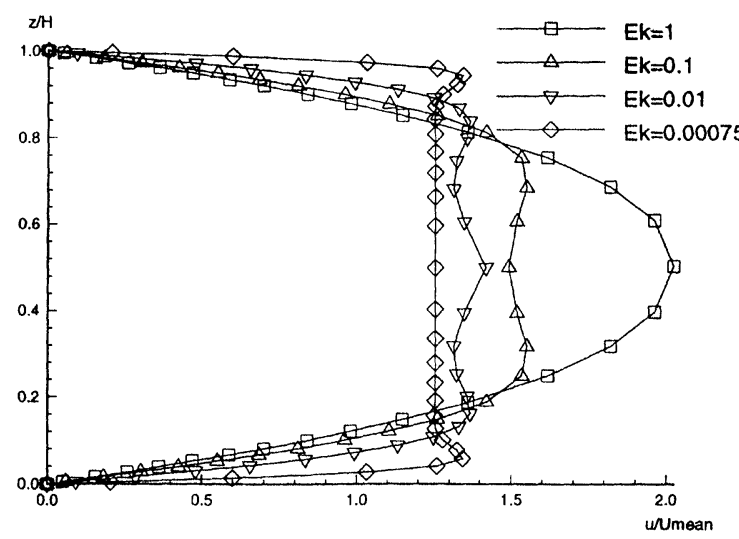

b)

FIGURE 6 Axial velocity profile in a rotating square duct for $\operatorname{Re}=500$ : (a) along the horizontal centerline of the duct; (b) along the vertical centerline of the duct.

the previous values, that is, Coriolis force has a substantial effect on the axial velocity flow profile.

Figure 7 shows the change of axial velocity with Rossby number when the convective inertia is small $($ Ro $=1,2)$ the secondary flow has two vortices. However when the Rossby number is 5, 10 and 20, the flow shows the feature of four vortices, which reveals three peaks of the axial velocity in the vertical centreplane. Therefore the effect of convective inertia is large; the flows have unstable region with a four-vortex.

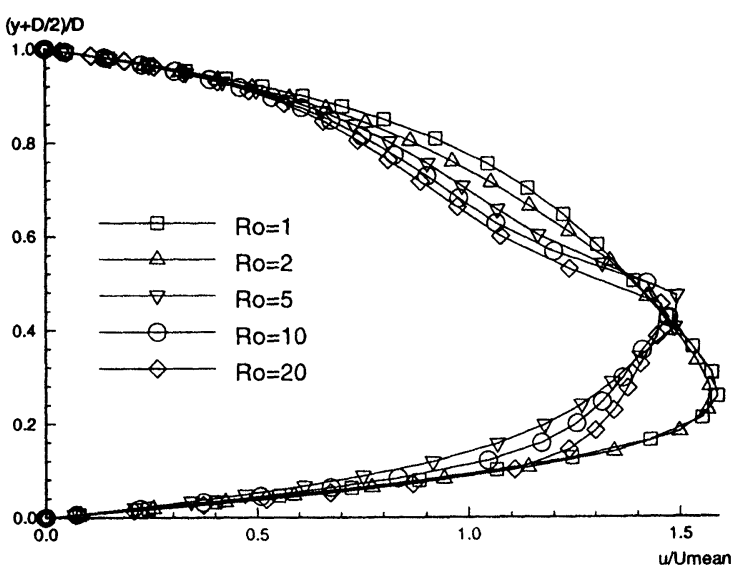

a)

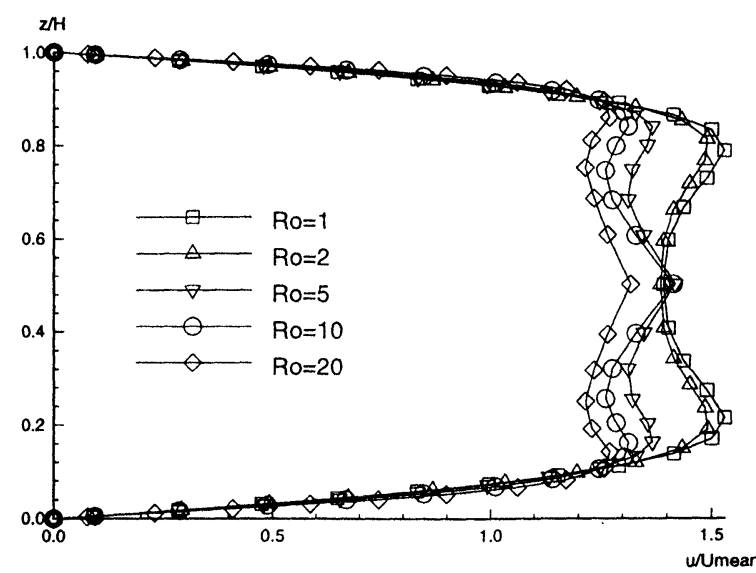

b)

FIGURE 7 Axial velocity profile in a rotating square duct for $E k=0.01$ : (a) along the horizontal centerline of the duct; (b) along the vertical centerline of the duct.

\subsection{Curved Duct}

The problem of the incompressible laminar flow in a curved square duct with a constant rotation is solved by the numerical method. In this case, the phenomena in a curved duct are influenced by the centrifugal force as well as the Coriolis force. This curved duct rotates about an axis parallel to the axial direction of the entrance region as shown in Fig. 2. In the curved region, the flow is affected by a centrifugal force and a Coriolis force as well. Because the centrifugal force is strong but the 
Coriolis force is weak at the Reynolds number of 500 , a secondary flow dominated by the centrifugal force appears as shown in Fig. 8. But the secondary flow becomes asymmetric with the increasing of the rate of rotation, since Coriolis force becomes stronger. However in the downstream region, the secondary flow subject to the Coriolis force is formed, since only the Coriolis force affects the flow. But the secondary flow of the downstream region becomes slightly asymmetric although the influence for the flow of the curved region are getting reduced as the flow goes to downstream. Of course if the length of the duct is long enough to reduce the effect of the centrifugal force, the secondary flow will become symmetric again Figure 9 shows the secondary flow patterns at

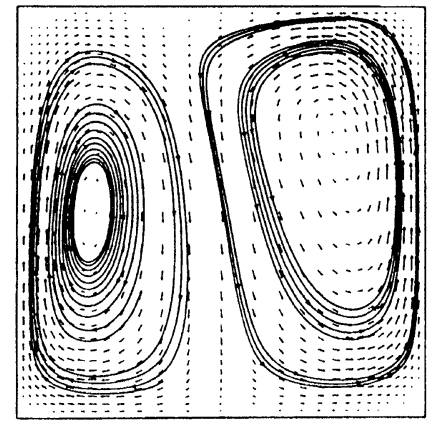

$\mathrm{Ek}=0.1$

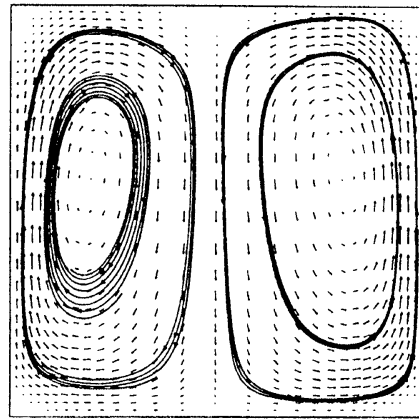

$\mathrm{Ek}=0.01$

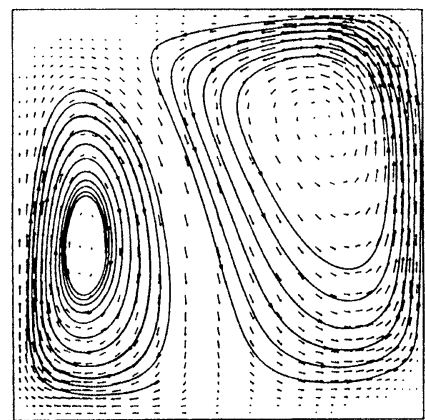

$\mathrm{Ek}=0.005$

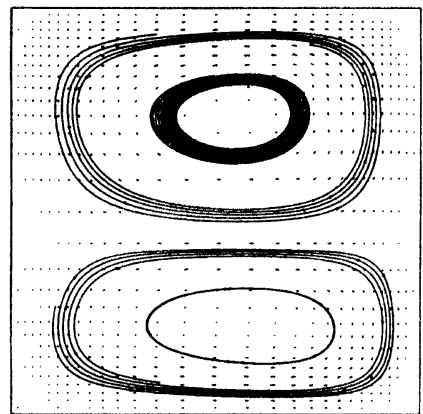

$\mathrm{Ek}=0.1$

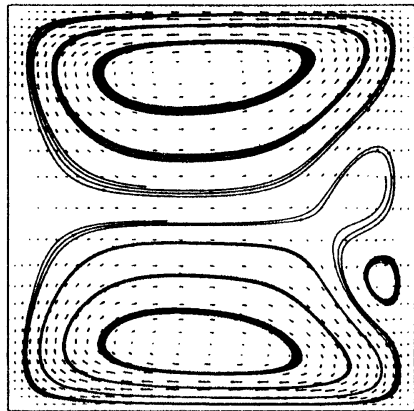

$\mathrm{Ek}=0.01$

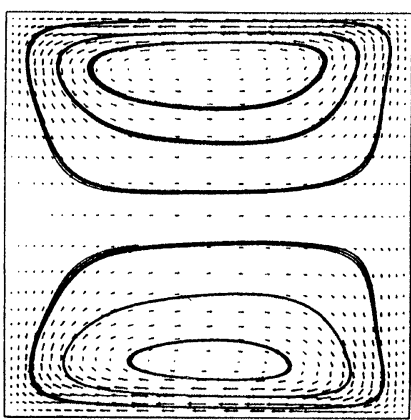

$\mathrm{Ek}=0.005$
FIGURE 8 Secondary flow in curved region for three Ekman number at $\mathrm{Re}=500$
FIGURE 9 Secondary flow in the downstream region for three Ekman number at $\mathrm{Re}=500$. 
the exit plane at difference Ekman numbers. The flow features at the exit plane shown in Fig. 9 are similar to those of the rotating straight duct at $\mathrm{Re}=500$.

\section{CONCLUSION}

A numerical study of the flow in the rotating straight duct and the rotating curved square duct has been conducted using the SIMPLE algorithm. At the straight square duct, the secondary flow consists of the type of double-vortex configuration and the axial velocity is nearly symmetric when the convective inertia is weak. And the axial velocity profile flattens as the rate of rotation increases. But for the strong convective inertia, a double-vortex secondary flow appears in the transverse planes of the duct for weak rotation rates but as the speed of rotation increases, the secondary flow is shown to be splitted into an asymmetric configuration with four vortices. If the rotation rates are increased further, the secondary flow restabilizes to a asymmetric double-vortex configuration. And the axial velocity profile is distorted by the secondary flow. When the rotation rate increases, the axial velocity along the vertical direction of the duct flattens and finally that profile has uniform profile in the core region.

In the curved square duct, the flow in the duct is more complicated by the interaction of the Coriolis force and the centrifugal force. In the curved region, the centrifugal force is dominant but in the downstream region, the Coriolis force has a noticeable effect on the flow. When the rate of rotation increases, an asymmetric secondary flow is formed in the curved region. But the flow in the downstream region is restored to the flow of a rotating straight duct.

\section{Acknowledgment}

This research was partially supported by School of Environmental Engineering, POSTECH. Their cooperation is gratefully acknowledged.

\section{NOMENCLATURE}

$\begin{array}{ll}D & \text { duct width }(\mathrm{m}) \\ \mathrm{Ek} & \text { Ekman number, } \mathrm{Ek}=\nu / \vec{\Omega} D^{2} \\ H & \text { duct height }(\mathrm{m}) \\ L & \text { straight duct length }(\mathrm{m}) \\ p & \text { pressure }\left(\mathrm{N} / \mathrm{m}^{2}\right) \\ P & \text { modified pressure, } P=p-\frac{1}{2} \rho \Omega^{2}\left(x^{2}+y^{2}\right) \\ P^{*} & \text { dimensionless modified pressure } \\ \vec{r} & \text { position vector }(\mathrm{m}) \\ \operatorname{Re} & \text { Reynolds number, } U D / \nu \\ \operatorname{Ro} & \text { Rossby number, } \mathrm{Ro}=U / \vec{\Omega} D \\ u_{\max } & \text { maximum } x \text {-direction velocity } \\ U & \text { axial mean velocity }(\mathrm{m} / \mathrm{s}) \\ v_{\max } & \text { maximum } y \text {-direction velocity } \\ \vec{V} & \text { velocity vector }(\mathrm{m} / \mathrm{s}) \\ \vec{V}^{*} & \text { dimensionless velocity vector } \\ x, y, z & \text { coordinate system } \\ \nu & \text { kinematic viscosity }\left(\mathrm{m}^{2} / \mathrm{s}\right) \\ \rho & \text { density }\left(\mathrm{kg} / \mathrm{m}^{3}\right) \\ \vec{\Omega} & \text { angular velocity }(\mathrm{rad} / \mathrm{s})\end{array}$

\section{References}

Barua, S.N. (1954) Secondary flow in a rotating straight pipe, Proceedings of the Royal Society of London A, 227, $133-139$.

Benton, G.S. (1956) The effect of the earth's rotation on laminar flow in pipes, Journal of Applied Mechanics, 23, $123-127$.

Hart, J.E. (1971) Instability and secondary motion in a rotating channel flow, Journal of Fluid Mechanics, 45, 341-351.

Johnston et al. (1972) Effects of spanwise rotation on the structure of two-dimensional fully developed turbulent channel flow, Journal of Fluid Mechanics, 56, 533-557.

Lezius, D.K and Johnston, J.P. (1976) Roll-cell instabilities in rotating laminar and turbulent channel flows, Journal of Fluid Mechanics, 77, 153-175.

Speziale, C.G. (1982) Numerical study of viscous flow in rotating rectangular ducts, Journal of Fluid Mechanics, 122 , 251-271.

Kheshgi, H.S. and Scriven, L.E. (1985) Viscous flow through a rotating square channel. Physics of Fluids, 28, 2968-2979. 


\section{ait \\ ENERGY MATERIALS}

M A N E Y publishing

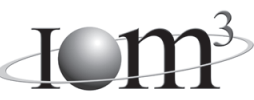

\section{Materials Science \& Engineering for Energy Systems}

Maney Publishing on behalf of the Institute of Materials, Minerals and Mining

The Institute of Materials, Minerals \& Mining

Economic and environmental factors are creating ever greater pressures for the efficient generation, transmission and use of energy. Materials developments are crucial to progress in all these areas: to innovation in design; to extending lifetime and maintenance intervals; and to successful operation in more demanding environments. Drawing together the broad community with interests in these areas, Energy Materials addresses materials needs in future energy generation, transmission, utilisation, conservation and storage. The journal covers thermal generation and gas turbines; renewable power (wind, wave, tidal, hydro, solar and geothermal); fuel cells (low and high temperature); materials issues relevant to biomass and biotechnology; nuclear power generation (fission and fusion); hydrogen generation and storage in the context of the 'hydrogen economy'; and the transmission and storage of the energy produced.

As well as publishing high-quality peer-reviewed research, Energy Materials promotes discussion of issues common to all sectors, through commissioned reviews and commentaries. The journal includes coverage of energy economics and policy, and broader social issues, since the political and legislative context influence research and investment decisions.

\section{CALL FOR PAPERS}

Contributions to the journal should be submitted online at http://ema.edmgr.com

To view the Notes for Contributors please visit: www.maney.co.uk/journals/notes/ema

Upon publication in 2006, this journal will be available via the Ingenta Connect journals service. To view free sample content online visit: www.ingentaconnect.com/content/maney

For further information please contact:

Maney Publishing UK

Tel: +44 (0)113 2497481 Fax: +44 (0)1132486983 Email: subscriptions@maney.co.uk

or

Maney Publishing North America

Tel (toll free): 8662975154 Fax: 6173546875 Email: maney@maneyusa.com

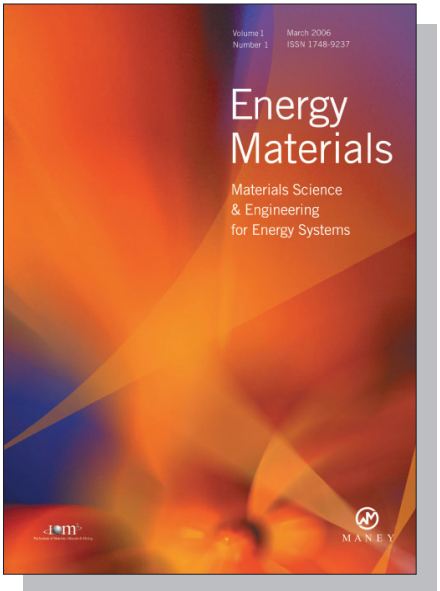

EDITORS

Dr Fujio Abe

NIMS, Japan

Dr John Hald, IPL-MPT, Technical University of Denmark, Denmark

Dr R Viswanathan, EPRI, USA

\section{SUBSCRIPTION INFORMATION}

Volume 1 (2006), 4 issues per year

Print ISSN: 1748-9237 Online ISSN: 1748-9245

Individual rate: $£ 76.00 / U S \$ 141.00$

Institutional rate: $£ 235.00 /$ US $\$ 435.00$

Online-only institutional rate: $£ 199.00 / U S \$ 367.00$

For special $\mathrm{IOM}^{3}$ member rates please email

subscriptions@maney.co.uk

\section{For further information or to subscribe online please visit www.maney.co.uk}



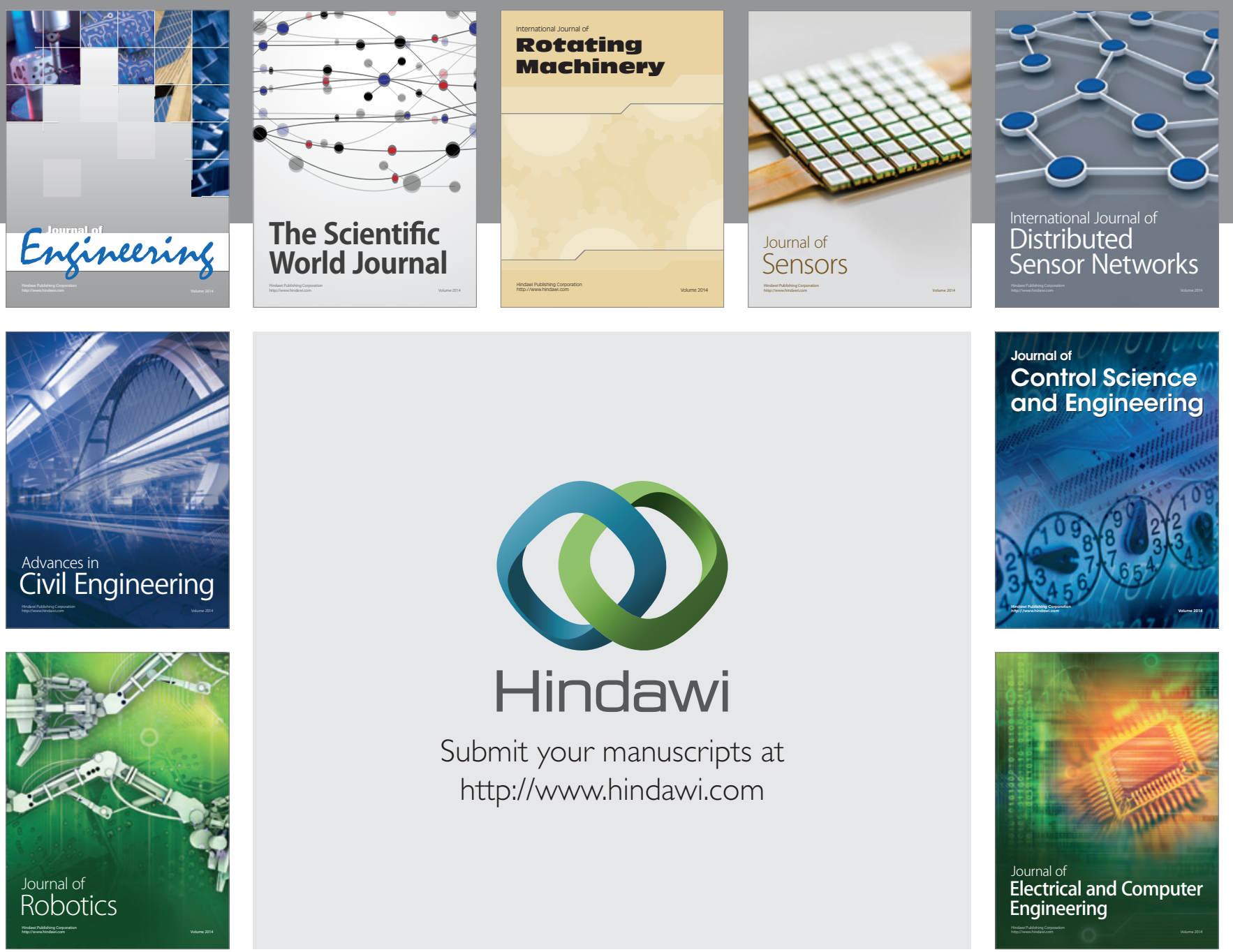

Submit your manuscripts at

http://www.hindawi.com
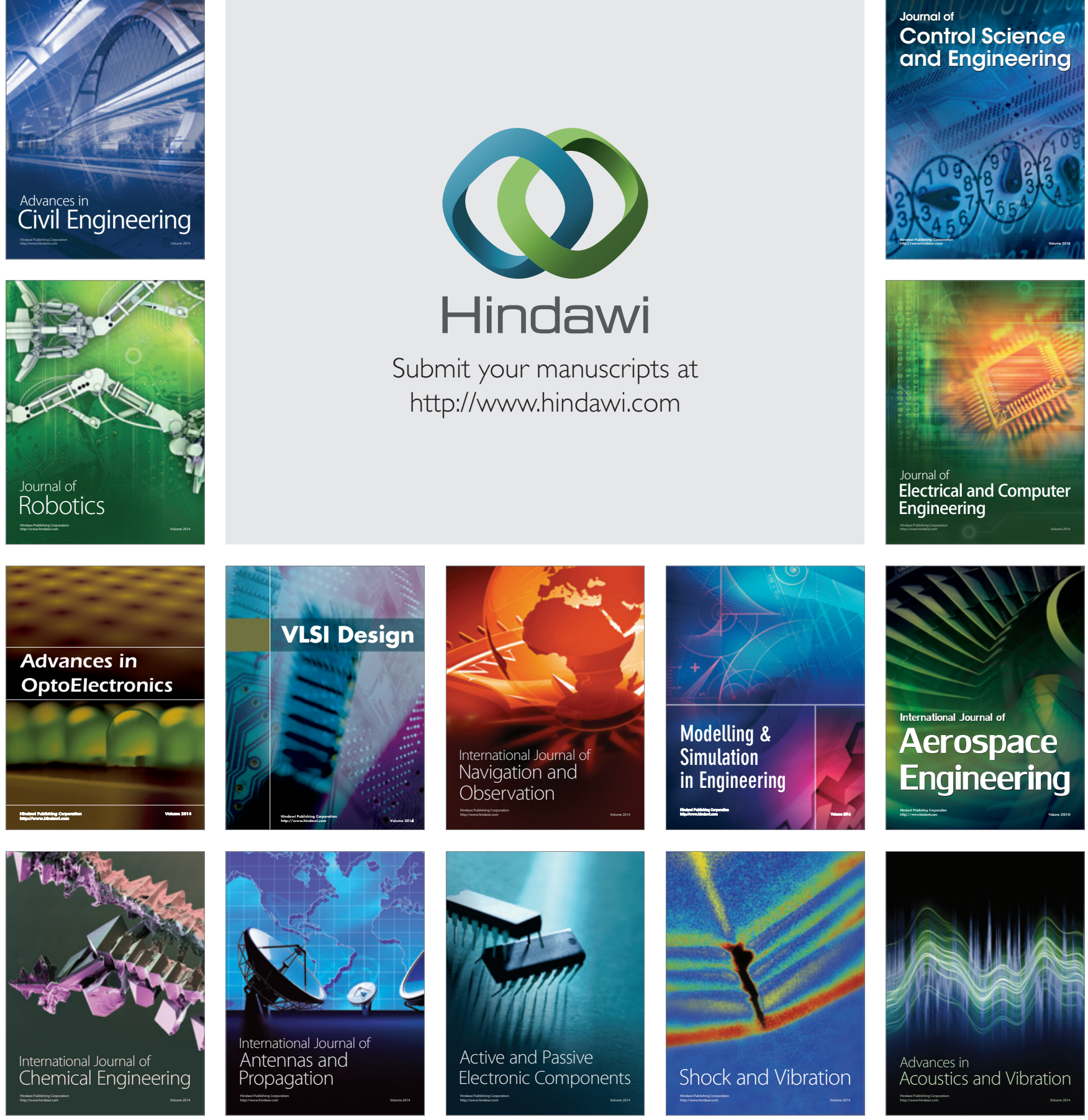